\title{
Automation of Political Biases DETECTION USING MACHINE LEARNING
}

\author{
Bill Zheng \\ Webb School of California, USA
}

\begin{abstract}
In the current political climate, mass media was depicted as highly divisive and inaccurate while many cannot efficiently identify its bias presented in the news. Using research regarding keywords in the current political environment, we have designed an algorithm that detects and quantifies political, opinion, and satirical biases present in current day articles. Our algorithm makes use of scipy'ssklearn linear regression model and multiple regression model to automatically identify the bias of a news article based on a scale of 0 to 3 (-3 to 3 in political bias detection) to automatically detect the bias presented in a news source. The usage of this algorithm on all three segments, politics, opinion, and satire has been proven effective, and it enables an average reader to accurately evaluate the bias in a news source.
\end{abstract}

\section{INTRODUCTION}

Media-processed news has become the predominant means in which Americans consume current information. Despite its presence in almost every facet of American life, many Americans today have expressed their discontent with the contemporary news service. With more than $62 \%$ of the Americans expressing that the mainstream media is biased and more than $80 \%$ of the Americans expressing that their news sources are biased, many set out to combat this polarization of news media in politics. Biases within a news source can be extremely harmful to the audience as misformation can produce harm at both a physical and a mental level. Millions of dollars have been spent on keeping the news impartial or detect any possible bias within the news, and this topic became more significant as we entered the present due to the polarization of news resources. An automated bias detection algorithm can not only save time for an average reader to identify the underlying biases of a news source, but it can also benefit the public by giving them more clarity in acquiring information and making rational decisions in the future.

While the issue of bias within the mainstream media was pervasive, many of the times readers were asked to identify the biases themselves as the media does not provide any insights on its own biases. Sometimes third-party fact-checkers such as Associated Press will conduct factchecking by themselves, but that method was limited in its usage. One study done by Budak, Goel, and Rao [1] indicates that the bias of each news source can be classified as an ndimensional vector and the computer can learn the bias of the vector by checking the attitude of the article on a certain topic. However, this study did not conclusively determine how an individual article presents its bias, instead, it gives us a holistic perspective on what biased is a news source. In the study, each of the data points gathered by the researchers was used to calculate the overall bias of a company instead of the bias of a given article, and that could be proven ineffective. Another practical problem is that this application does not provide a causal or correlational relationship between individual articles and its publishers and its context, which, if not used accurately, could produce misinformation and cannot be more complex than just indicating the general direction of the research.

David C. Wyld et al. (Eds): MLNLP, BDIoT, ITCCMA, CSITY, DTMN, AIFZ, SIGPRO - 2020

pp. 175-181, 2020. CS \& IT - CSCP 2020

DOI: $10.5121 /$ csit.2020.101218 
In this paper, we follow the same line of research by Hamborg, Donnay, and Gipp [2]. Our goal is to use the semantics given in the news article to formulate the bias of each article. Our method is inspired by Hamborg, Donnay, and Gipp as they introduced both the source of the news and the semantics of the news together. First, we analyzed the source of the news through the means of identifying each keyword -- that is both a topic revolving around politics and adjectives in a specific manner in which positively and negatively describing things in one manner would result in a specific result from the multiplier. Using linear regression, one would get the results of bias/opinion bias/satire bias in the form of the slope of the best-fit regression, which will then be compared with the consensus to adjust the final value. This training will be iterated through many samples, and this ensures the accuracy of detecting biases that are subtle or satirical contents. Both the source of the news and the semantics will be combined in this method, which will also provide a holistic perspective on the biases presented in the news.

We have tested three distinct scenarios using a total of 8 different articles from 8 distinct news sources that are known to have a certain bias, opinion, and satirical tone. We dissect them into two segments with one of them being less opinionated/ biased/ satirical tone than the other. We have done this method two times, one with 100 pieces of 15 -word segments and one with 200 pieces of 15-word segments in training. Both of these experiments have shown varying degrees of success in detecting various forms of biases in news reports compared to the consensus. After training with 200 pieces of 15 -word segments, our accuracy in terms of convergence of a specific piece of text has improved by $15 \%$ in evaluating the total range of the articles compared to the consensus.

Section 2 presents the challenges met in the processing of experimenting with the algorithm and ways of circumventing around it. Section 3 focuses on the solution and walks through its process of creating it while corresponding to each section with section 2. Section 4 presents details, results, and analysis of the experiment in section three, which is followed by presenting works that helped the creation of this paper in section 5. The section concludes the paper while giving future insights into similar projects.

\section{Challenges}

The biggest challenge of working on this project entails the inclusion of internal biases. The politics involved within the media culture is tremendous, and it is completely subjective and it is up to the interpretation of the audience. One of the more significant examples is the difference between the worldview between European politics and American politics. In general, Americans are more right-leaning than Europeans given the same ideological tenets of a politician. It should also be noted that defining biases is purely subjective as a right-winger is more tolerant towards right-wing content than a left-winger. Therefore, it is hard to find the consensus and to check the accuracy of a given article based on the information produced.

The second challenge regards dealing with the erratic nature of news reporting. Given that there are multiple news reporters and journalists with a wide range of ideological beliefs, it is hard to keep a track of everything that they are reporting. For example, the Wall Street Journal has a wide variety of reporters and journalists with some leaning to the right and some leaning to the left, and it has a high discrepancy within the own firm. Therefore, one of the major challenges will be to classify the distribution of the biases within a single firm and to make it as accurate as possible.

The final challenge regarding this paper is finding the appropriate sources. While there were some papers automating the process of political bias detection, they are mostly limited to finding 
the biases of a certain keyword of a certain news source. It should also be noted that not many would attempt to write a thesis based on the two challenges presented above. Therefore, it would be harder than expected to find any credible resources to prove the effectiveness of this thesis and its experiment.

\section{Methodology/Solution}

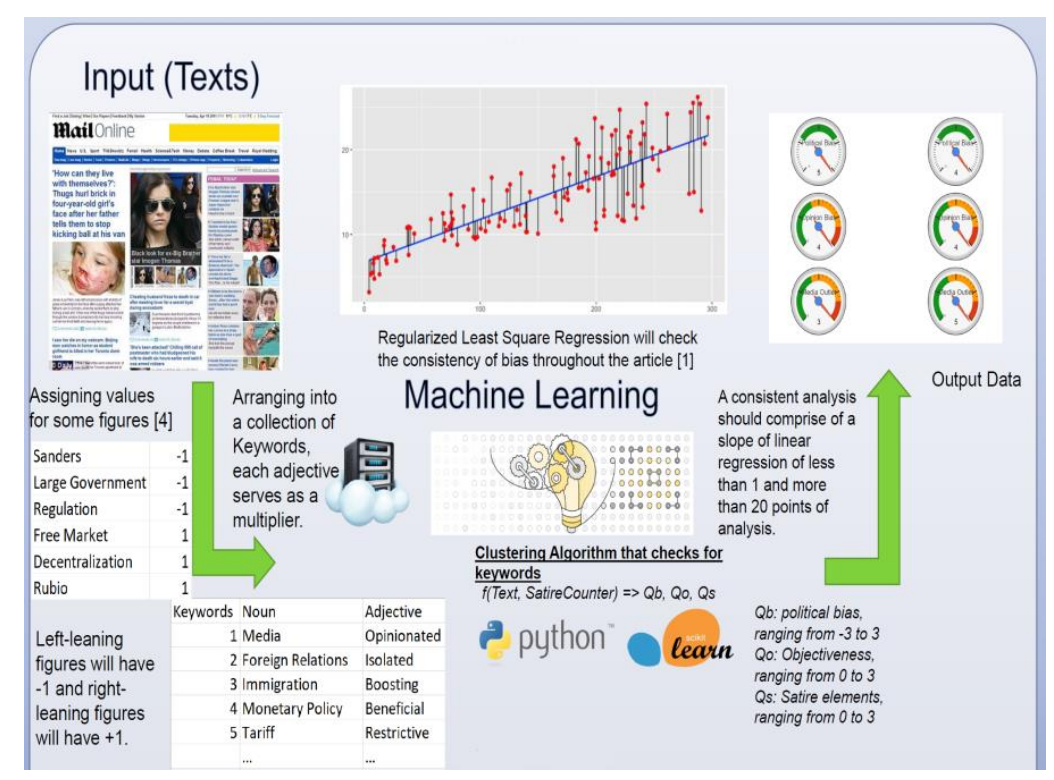

The system will first break the word segment into smaller samples based on the keyword dictionary provided. It will then use the dictionary to identify a numerical value for all of the word samples by giving a left-wing word a negative value and a right-wing word a positive value to correspond with their respective positions on a cartesian plane. It will then find any adjectives associated with such keywords, and it will multiply it with the adjective given with a positive value for a positive adjective and a negative value for a negative adjective. Using that, it will use regularized least square regression to check the apparent biases of the news by inserting the keywords into their original position as indicated by the numerical value, then it will compare itself with the sample already learned by the computer that has undergone the same procedure and has been given a numerical value for its biases to ensure its accuracy. 


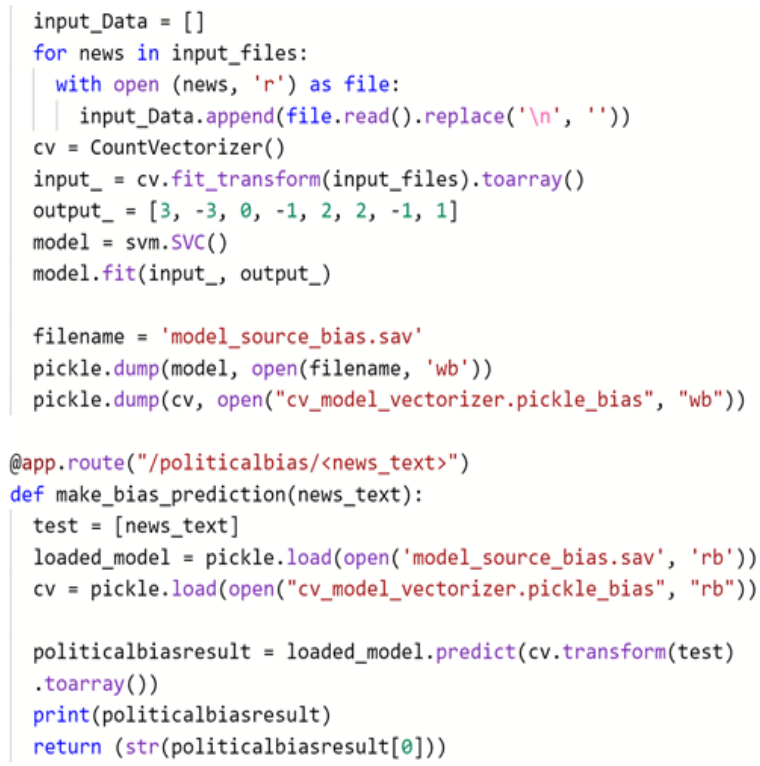

The code follows the same patterns as the steps discussed above: the computer will first detect relevant keywords and use a method of least regularized square regression to calculate the theoretical biases as described above, then it will match with the texts that have a similar set of keywords and compare their values. Data will be saved through pickle, and the real value will be sent out through methods of count vectorizer transforms. The final results will be printed and displayed in the dials below.

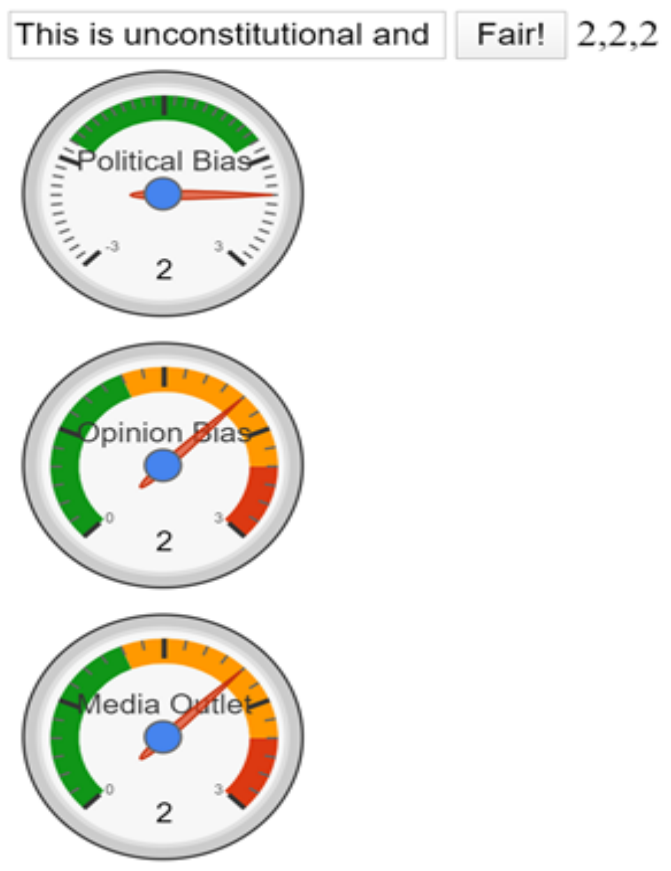

The first part of the algorithm attempts to quantify the biases using the linear regression model, then the linear regression will give an output and correlate it to the given value in the list. They will be fitted together and used in machine learning through a count vectorizer. 


\section{EXPERIMENTS/EvAluATION}

My solution strives to automate the detection of political bias within a piece of text, and the algorithm has guaranteed that it will generate an accurate result. My experiment includes using eight different articles with varying degrees of political, opinion, and satirical biases from 4 different sources. It totals up to around 2000 words, which will be fitting for the learning process.

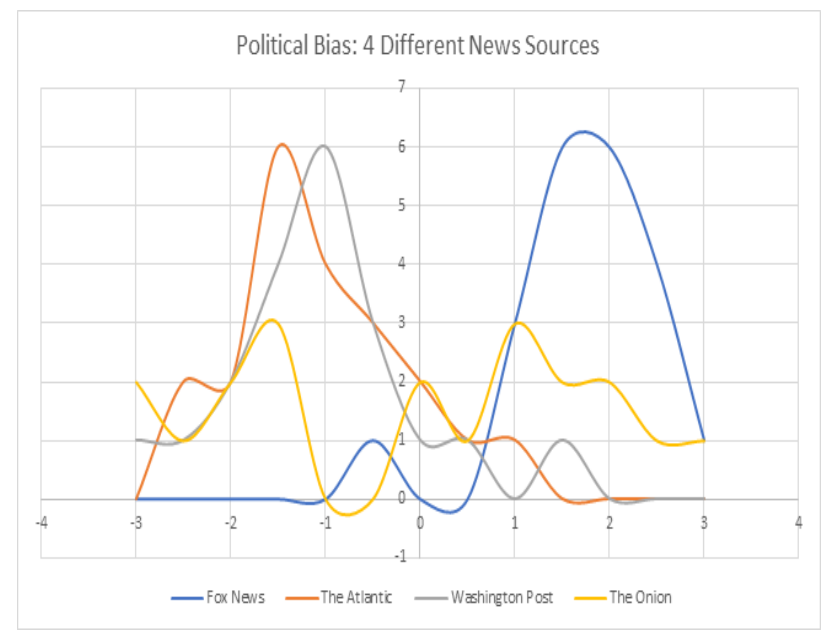

Numerical Average:

Fox News: 1.92

The Atlantic: -1.52

Washington Post: -0.98

The Onion: 0.22

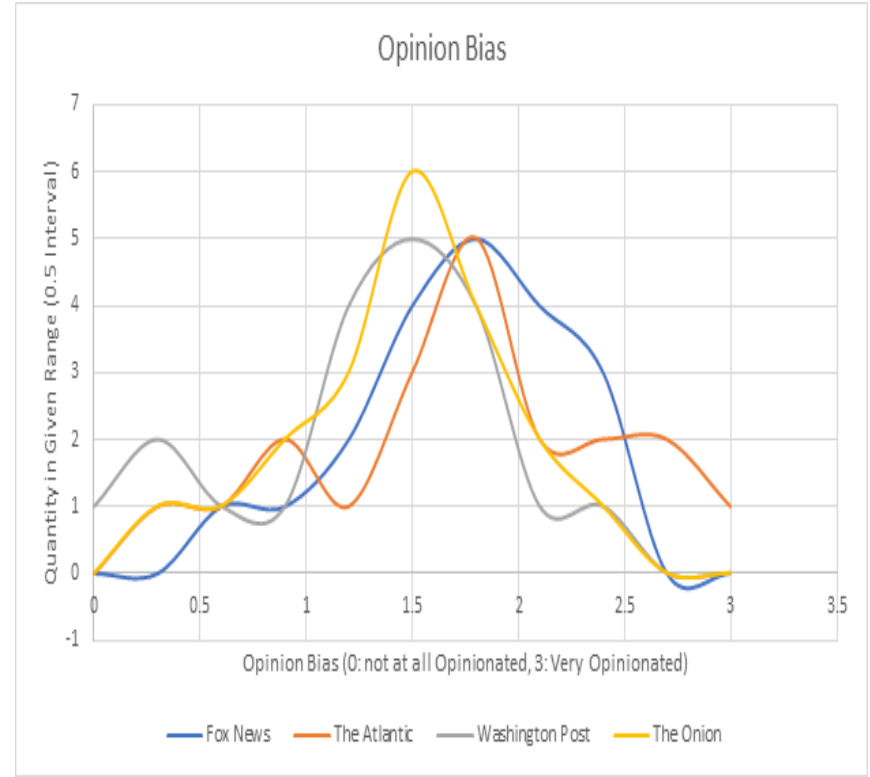

Numerical Average:

Fox News: 2.02

The Atlantic: 1.88

Washington Post: 1.51

The Onion: 1.44 


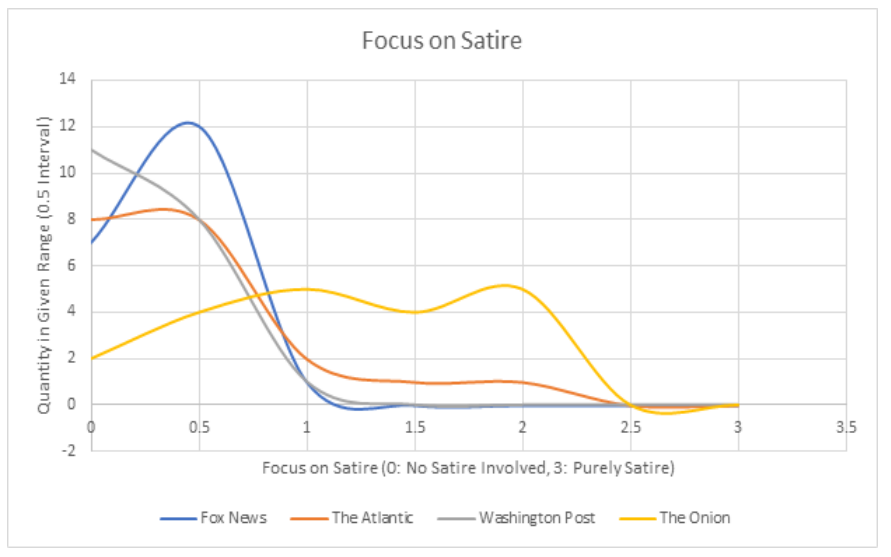

Numerical Average:

Fox News: 0.42

The Atlantic: 0.88

Washington Post: 0.30

The Onion: 1.88

The second experiment is designed to test the algorithm's susceptibility to change, and more specifically the susceptibility of change in keywords. In each of the 30 words segment, we had 5 keywords change throughout the entire passage randomly, and we tested the susceptibility to keyword change without changing the general meaning of the article.

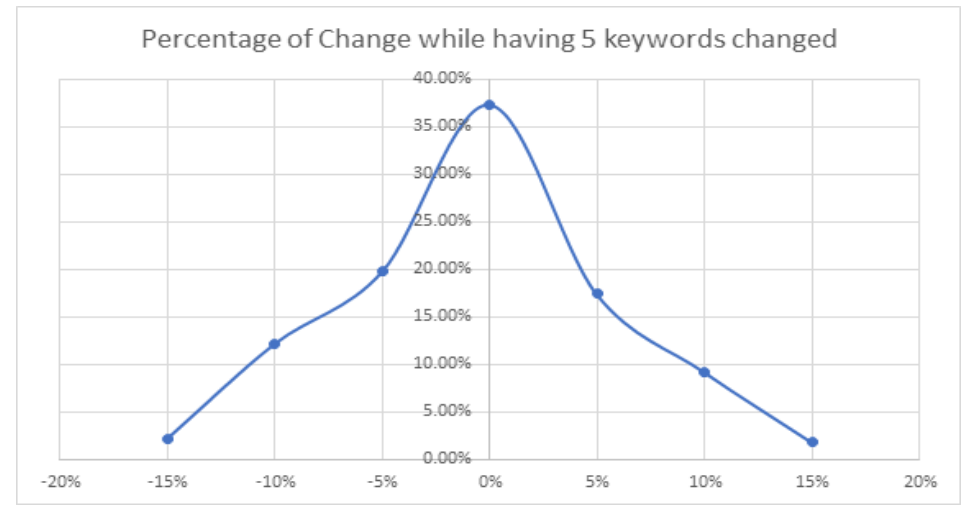

Compared to the general consensus, my results have shown that the twenty pieces gathered at random have shown great accuracy with each of them having less than $15 \%$ error. As of the changing keywords, despite it causing some major change in the results of the algorithm, calculations have shown that the standard deviation is around 4\%, which shows that this algorithm while facing some changes in the keywords section, are in fact less susceptible to the changes in them.

\section{RELATED WORK \& REFERENCES}

\section{Related work 1}

https://www8.gsb.columbia.edu/media/sites/media/files/JustinRaoMediaBias.pdf

This work is used to identify political biases within each news source by analyzing the semantics of each news source. My work expanded on this work by using the semantics and given values of 
a text to fit the biases of each text instead of each news source. I improved the ways this study went as I used a more holistic way to approach this issue and provided a more stable and accurate result.

\section{Related work 2}

https://link.springer.com/article/10.1007/s00799-018-0261-y

This source discussed the potential role that the news publisher could have on emanating biases, and it also discussed how to use machine learning to detect biases by giving each way of using different semantics a fixed value. I believe this is a great foundation for my work as it also provided much of the materials needed for constructing my machine learning algorithm. My algorithm focuses less on the source, and it is more efficient as it simply combines two algorithms which have been laid foundations on.

\section{Related work 3}

Hainmueller, J., \& Hazlett, C. (2014). Kernel Regularized Least Squares:

Reducing Misspecification Bias with a Flexible and Interpretable

Machine Learning Approach.

Political Analysis, 22 (2), 143168.

doi:10.1093/pan/mpt019

Compared to the two algorithms discussed above, the algorithm introduced in this study focuses on a specific way of regression: Kernel regularized least squares. It helped me to provide a more accurate reading on the regression, and I used his work as a part of my algorithm. However, with the combined algorithm, I can create more applications for this mean of regression and put it into great use with great accuracy.

\section{CONCLUSION AND FUTURE WORK (400+ WORDS)}

In this paper, we develop a system which uses the semantics given in the news article to detect and quantify political, opinion, and satirical biases present in current day articles. Firstly, the system will first break the word segment into smaller samples based on the keyword dictionary provided. Then, it will identify a numerical value for all of the word samples by giving a left- wing word a negative value and a right-wing word a positive value to correspond with their respective positions. The results of bias/opinion bias/satire bias will be obtained in the form of the slope of the best-fit regression through linear regression. For the data training, it will be iterated through many samples, and this ensures the accuracy of detecting biases that are subtle or satirical contents. Both the source of the news and the semantics will be combined in this method, which will also provide a holistic perspective on the biases presented in the news.

In future, we will improve our model and make sure that the bias/opinion bias/satire bias can reflect the real case precisely.

(C) 2020 By AIRCC Publishing Corporation. This article is published under the Creative Commons Attribution (CC BY) license. 